\title{
Funding cuts put pressure on peer review
}

Capri, Italy. Cuts in research funding, and the resulting increase in competition for funds between scientists, have exposed the shortcomings of the peer review system. But it remains the best system in operation, according to an international 'consensus' conference on research assessment held in Italy last week.

The conference, on the island of Capri, near Naples, was held under the auspices of an informal group of representatives from G7 countries. The scientists and policymakers from three continents who attended agreed that the peer review system needs to be improved to fit new circumstances.

They also agreed that objective criteria, such as citation data, can be helpful in assessing research departments, but should only be used very carefully when assessing individuals. This was particularly true for the academic community of the host country, Italy, which is struggling to institute wide-ranging reforms, but has little confidence in its current procedures for reviewing grant applications and for career promotion.

Much of the current pressure on peer review results from oversubscription to research programmes, with the result that the success rates of applications have fallen to levels widely considered unhealthy. "This not only strains the time of reviewers, it also strains the concept that the system is operating fairly," said Susan Cozzens, a policy officer at the US National Science Foundation.

Cozzens said low success rates tend to favour solid, but low-risk, applications. Others argued that this situation also undermines the value of peer review, as the difficulty of choosing objectively between closely-ranked top-quality applications can make the selection of successful projects little more than a lottery.
Several participants pointed out that grant agencies are acutely aware of the strain on reviewers. Many leading scientists are unhappy with the drudgery of wading through stacks of low-quality grant applications, and frustrated that applications they rate highly remain unfunded because of lack of money.

Some funding agencies are experimenting with pre-screening applications to weed out 'no-hopers' at an early stage. But prescreening, with its spectre of 'assessment by administrators', is unpopular in much of the scientific community, and can add to a general perception of unfairness.

The conference agreed that confidence in the fairness of a peer-review system can be increased by the appropriate use of bibliometric data, such as publication rates and 'impact factors', which measure citation rate. But participants acknowledged that such data should be used carefully, particularly in assessing individuals, and should never replace human judgement entirely.

It is a sensitive issue. Many researchers feel that the mathematical detachment of citation data implies a certainty that is attractive, but is not always warranted. The conference agreed that such data can be misleading, for example on multi-author papers, as a laboratory chief or supplier of biological material may routinely add his or her name to papers to which they have made no scientific contribution.

Indeed the conference was told by Eugene Garfield that citation indices had never been intended for evaluation. Garfield, who developed the concept of impact factors as a measure of a journal's influence, is founder, and now emeritus chairman, of the Institute for Scientific Information in Philadelphia.

Garfield said that he had been widely depicted as "a Frankenstein" whose monster - the impact factor of an individual paper - had an enormous potential to be misused "in the hands of uninformed users".

Pressure to rely heavily on publicationbased indicators is particularly strong in Italy, where many grant-giving agencies and charities distribute small amounts of money evenly, to avoid upsetting unsuccessful

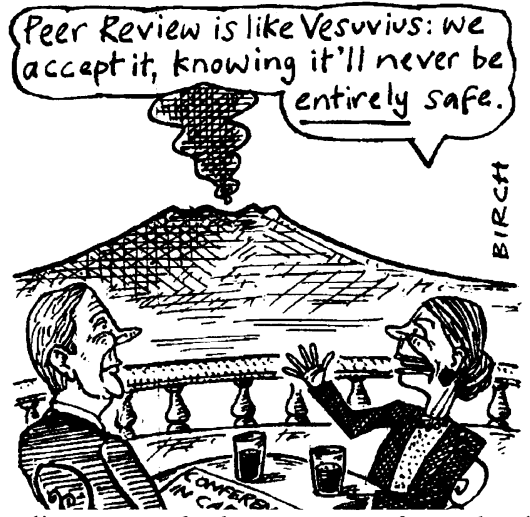

applicants, and the system of academic promotion through national competitions, or concorsi, is widely criticized.

Many Italian scientists feel that the lack of formal selection criteria has allowed personal contacts to play too important a role in the allocation of professorships and assistant professorships. Those at the conference described how the academic community is split over how much weight should be given to citation data in assessing an individual's research performance.

Some feel that such data should at least be used to eliminate individuals with low citation ratings at an early stage. Complex formulae for weighting the value of each publication have been suggested as ways of compensating for the weaknesses of citation data - for example, scaling down the impact factor of a multi-author paper or a review, which normally have higher impact factors than a research paper.

Proponents argue that such formulae would allow citation data to be used fairly. But others fear that excessive reliance on such data could allow the merits of a firstclass scientist, who has published little, to be overlooked.

The conference agreed in one of a series of formal statements that citation indices should only be used as one of a number of different performance indicators. It said that an assessment committee should be able to give a high rating to a 'low impact' individual - and vice versa - provided that it is able to give an open justification of its decision.

The conference also agreed that citation indices are too crude to distinguish between closely-ranked grant applications. As a result, complex formulae intended to quantify the merit of individual publications are unnecessary.

Alison Abbott 